I. Yu. Kotova ${ }^{a}$, A. A. Savina ${ }^{a}$, A. I. Vandysheva ${ }^{\text {b }}$, D. A. Belov ${ }^{c}$, S. Yu. Stefanovich ${ }^{c}$

${ }^{a}$ Baikal Institute of Nature Management, Siberian Branch, Russian Academy of Sciences, 6 Sakhyanova St., Ulan-Ude, 670047, Buryat Republic, Russian Federation ${ }^{b}$ Buryat State University,

24 a Smolin St., Ulan-Ude, 670000, Buryat Republic, Russian Federation

'Lomonosov Moscow State University, Leninskie Gory, 1, Moscow 119991, Russian Federation ${ }^{\star}$ E-mail: alex551112@mail.ru

\title{
Synthesis, crystal structure and electrophysical properties of triple molybdates containing silver, gallium and divalent metals
}

A possibility of the triple molybdates formation with both NASICON-like and $\mathrm{NaMg}_{3} \operatorname{In}\left(\mathrm{MoO}_{4}\right)_{5}$ structures in the $\mathrm{Ag}_{2} \mathrm{MoO}_{4}-\mathrm{AMoO}_{4}-\mathrm{Ga}_{2}\left(\mathrm{MoO}_{4}\right)_{3}(A=\mathrm{Mn}, \mathrm{Co}, \mathrm{Zn}$, $\mathrm{Ni})$ systems was studied by powder $\mathrm{X}$-ray diffraction analysis. It was established that NASICON-like phases $\mathrm{Ag}_{1-x} A_{1-x} \mathrm{Ga}_{1+x}\left(\mathrm{MoO}_{4}\right)_{3}$ are not formed. The triple molybdates $\mathrm{AgA}_{3} \mathrm{Ga}\left(\mathrm{MoO}_{4}\right)_{5}(A=\mathrm{Mn}, \mathrm{Co}, \mathrm{Zn})$ isostructural to triclinic $\mathrm{NaMg}_{3} \mathrm{In}\left(\mathrm{MoO}_{4}\right)_{5}$ (sp. gr. $P \overline{1}, Z=2$ ) were synthesized and characterized. The structure of the obtained compounds was refined for $\mathrm{AgZn} 3 \mathrm{Ga}\left(\mathrm{MoO}_{4}\right)_{5}$ according to the powder data by the Rietveld method. The structure consists of $\mathrm{MoO}_{4}$ tetrahedra, couples of edge-shared $M(1) \mathrm{O}_{6}$ octahedra, and trimers of edge-shared $M(2) \mathrm{O}_{6-}, M(3)_{6-}$ and $M(4) 0_{6}$ octahedra, which are linked by the common vertices to form a 3D framework. High-temperature conductivity measurements revealed that the conductivity of $\mathrm{AgMn} 3 \mathrm{Ga}\left(\mathrm{MoO}_{4}\right)_{5}$ at $500^{\circ} \mathrm{C}$ reaches $10^{-2} \mathrm{~S} / \mathrm{cm}$, which is close to one of the known NASICON-type ionic conductors.

Keywords: triple molybdates; silver; gallium; solid-state synthesis; powder X-ray diffraction; Rietveld refinement; ionic conductivity.

Received: 10.10.2018. Accepted: 22.10.2018. Published: 31.10.2018.

(C) Kotova I. Yu., Savina A. A., Vandysheva A. I., Belov D. A., Stefanovich S. Yu., 2018

\section{Introduction}

A synthesis and studying of complex oxide compounds, the development of new materials with functionally significant properties based on those are among the main areas of the materials science. An important place in the study and obtaining of new phases with valuable physicochemical properties belongs to mo- lybdates, in particular triple ones, which are among the fastest-growing groups of complex oxide compounds containing a tetrahedral anion and three different cations. One of the largest families of these compounds is molybdates containing 1-, 2- and 3-charged cations. In particular, silver-containing NASICON-like phases 
$\mathrm{Ag}_{1-x} A_{1-x} R_{1+x}\left(\mathrm{MoO}_{4}\right)_{3}(A=\mathrm{Mg} \mathrm{Co}, R=\mathrm{Al}$; $A=\mathrm{Mg}, R=\mathrm{In})$ with different homogeneity range and triclinic $\operatorname{Ag} A_{3} R\left(\mathrm{MoO}_{4}\right)_{5}$ $(A=\mathrm{Mg}, R=\mathrm{Cr}, \mathrm{Fe}, \mathrm{Ga} ; A=\mathrm{Mn}, R=\mathrm{Al}$, $\mathrm{Cr}, \mathrm{Fe}, \mathrm{Sc}, \mathrm{In})$ having high ionic conductivity $\left(10^{-3}-10^{-2} \mathrm{~S} / \mathrm{cm}\right)$ are of interest. For a number of phases: $\operatorname{AgMg}_{3} R\left(\mathrm{MoO}_{4}\right)_{5}$ $(R=\mathrm{Cr}, \mathrm{Fe}), \mathrm{AgMn}_{3}^{\mathrm{II}}\left(\mathrm{Mn}_{0.26}^{\mathrm{III}} \mathrm{Al}_{0.74}\right)$ $\left(\mathrm{MoO}_{4}\right)_{5}, \mathrm{Ag}_{0.90} \mathrm{Al}_{1.06} \mathrm{Co}_{2.94}\left(\mathrm{MoO}_{4}\right)_{5}$ and $\mathrm{AgFe}{ }_{3} \mathrm{Fe}^{\mathrm{III}}\left(\mathrm{MoO}_{4}\right)_{5}$ single crystals were

\section{Experimental}

The initial materials were simple molybdates of silver, manganese, cobalt, zinc, nickel, $\mathrm{MoO}_{3}$ and $\mathrm{Ga}_{2} \mathrm{O}_{3}$ (reagent grade).

$\mathrm{Ag}_{2} \mathrm{MoO}_{4}$ and molybdates of divalent metals were obtained by the step annealing of stoichiometric mixtures of $\mathrm{AgNO}_{3}$ (analytical grade), $\mathrm{MnO}, \mathrm{Co}\left(\mathrm{NO}_{3}\right)_{2} \cdot 6 \mathrm{H}_{2} \mathrm{O}$, $\mathrm{ZnO}, \mathrm{MoO}_{3}$ (all chemically pure), $\mathrm{NiO}$ (reagent grade) at $350-450^{\circ} \mathrm{C}\left(\mathrm{Ag}_{2} \mathrm{MoO}_{4}\right)$, $400-750^{\circ} \mathrm{C}\left(\mathrm{MnMoO}_{4}\right), \quad 300-700^{\circ} \mathrm{C}$ $\left(\mathrm{CoMoO}_{4}\right), \quad 500-700^{\circ} \mathrm{C} \quad\left(\mathrm{ZnMoO}_{4}\right)$, $450-750{ }^{\circ} \mathrm{C}\left(\mathrm{NiMoO}_{4}\right)$ in the air with intermittent grindings every 15 hours for better sample homogenization. Power $\mathrm{X}$-ray diffraction (PXRD) patterns of the prepared compounds do not contain reflections of starting or impurity phases. PXRD and thermal characteristics of all prepared compounds agree well with corresponding data reported in [10-15].

Sample compositions $\mathrm{Ag}_{1-x} A_{1-x} \mathrm{Ga}_{1+x}$ $\left(\mathrm{MoO}_{4}\right)_{3}(0 \leq x \leq 0.7, \Delta x=0.1)$ and $\mathrm{Ag} A_{3} \mathrm{Ga}\left(\mathrm{MoO}_{4}\right)_{5}$ were prepared by the annealing of appropriate stoichiometric mixtures of $\mathrm{Ag}_{2} \mathrm{MoO}_{4}, \mathrm{AMoO}_{4}, \mathrm{MoO}_{3}$ and $\mathrm{Ga}_{2} \mathrm{O}_{3}$. The initial mixtures were annealed starting at $300^{\circ} \mathrm{C}$ followed by raising the temperature by $20-50^{\circ} \mathrm{C}$ (in some cases, $5-10^{\circ} \mathrm{C}$ ) with intermittent grindings every 20-30 hours for sample homogenization. The calcination time at each temperature was $30-70 \mathrm{~h}$. The phase composition of the obtained and their crystal structures were determined [1-9].

The purpose of this work is to study the possibility of forming triple molybdates $\mathrm{Ag}_{1-x} A_{1-x} \mathrm{Ga}_{1+x}\left(\mathrm{MoO}_{4}\right)_{3}$ and $\mathrm{Ag}_{3} \mathrm{Ga}\left(\mathrm{MoO}_{4}\right)_{5}(A=\mathrm{Mn}, \mathrm{Co}, \mathrm{Zn}, \mathrm{Ni})$ and investigate crystal structure and electrophysical properties of the obtained compounds.

obtained products was controlled by the PXRD analysis before each increasing of the annealing temperature.

PXRD patterns were collected at room temperature on a Bruker D8 ADVANCE diffractometer using $\mathrm{Cu} \mathrm{Ka}$ radiation in the $2 \theta$ range from $5^{\circ}$ to $100^{\circ}$ with a step of $0.02076^{\circ}$. Possible impurity phases were checked by comparing their PXRD patterns with those in the Powder Diffraction File. The crystal structure refinement was carried out with the GSAS [16] program suite using PXRD data. Lattice parameters and individual scale factors were established, and five common peak-shape parameters of the pseudo-Voigt function (No. 2), one asymmetry parameter and one parameter for the zero-point correction were used to describe the powder patterns. The background level was described by a combination of 15-order Chebyshev polynomials. Isotropic displacement parameters (Uiso) were refined, and grouped by chemical similarity by used constrains.

Thermoanalytic studies were carried out on a STA 449 F1 Jupiter NETZSCH thermoanalyser (Pt crucible, heating rate of $10^{\circ} \mathrm{C} / \mathrm{min}$ in $\mathrm{Ar}$ stream).

Ceramic disks for dielectric investigations were prepared by the calcination of pressed powder at $600^{\circ} \mathrm{C}$ for $2 \mathrm{~h}$. The disks were of 9-10 $\mathrm{mm}$ in diameter and 
1-2 mm thick, the electrodes were deposited by painting the disk bases with colloid platinum followed by subsequent one hour annealing at about $580^{\circ} \mathrm{C}$. The direct current (DC) electric conductivity was measured with a V7-38 microammeter. To study the ion transfer, electrical conductivity was measured on an alternating current (AC) by the two-contact method

\section{Results and discussion}

\section{PXRD characteristics}

The presence of NASICON-like phases in the $\mathrm{Ag}_{2} \mathrm{MoO}_{4}-\mathrm{AMoO}_{4}-\mathrm{Ga}_{2}\left(\mathrm{MoO}_{4}\right)_{3}$ systems was determined according to PXRD analysis of samples $\mathrm{Ag}_{1-x} A_{1-x} \mathrm{Ga}_{1+x}\left(\mathrm{MoO}_{4}\right)_{3}$ $(0 \leq x \leq 0.7, \Delta x=0.1)$ which were annealed in the temperature range from $300^{\circ} \mathrm{C}$ to melting point. The final annealed temperature was $550-700^{\circ} \mathrm{C}$ and depended on both the composition of the reaction mixtures and the nature of the divalent metal. It was established that, despite the close values of the $\mathrm{Al}^{3+}(0.53)$ and $\mathrm{Ga}^{3+}$ (0.62 $\AA$ [17]) radii, gallium containing triple molybdates with NASICON-like structure, apparently, do not exist. All our attempts to obtain rhombohedral phases $\mathrm{Ag}_{1-x} A_{1-x} \mathrm{Ga}_{1+x}\left(\mathrm{MoO}_{4}\right)_{3}$ by solid state synthesis did not lead to a positive result, probably this is due to the low reactivity of gallium in the molybdate systems. Thus, the simple gallium molybdate $\mathrm{Ga}_{2}\left(\mathrm{MoO}_{4}\right)_{3}$ has not yet been obtained by ceramic technology, and only recently it was synthesized by the sol-gel method [18]. Besides, silver-gallium double molybdate is not synthesized either by ceramic technology or by co-precipitation. In [19] this compound was obtained by the calcining of mixtures of $\mathrm{AgNO}_{3}, \mathrm{Ga}_{2} \mathrm{O}_{3}, \mathrm{MoO}_{3}$ (in ratio $2: 1: 4)$ at $350-400^{\circ} \mathrm{C}$ for $8-10 \mathrm{~h}$, followed by cooling, homogenization, and the repeated 12-20 hours annealing at 500- in the frequency range $1 \mathrm{~Hz}-1 \mathrm{MHz}$ in the temperature range $25-560^{\circ} \mathrm{C}$ at the rate of $4^{\circ} \mathrm{C} / \mathrm{min}$ at both heating and cooling using a Novocontrol Beta-N impedance analyzer. The activation energy of electrical conductivity was calculated from the slope of the straight lines corresponding to the Arrhenius dependence in $\lg (\sigma T)-\left(10^{3} / T\right)$ coordinates.

$550^{\circ} \mathrm{C}$, but the PXRD data of the product are not given by the authors. It should be noted that in none of the later publications (including those of the same authors) additional information about this compound was found.

At the same time, in the $\mathrm{Ag}_{2} \mathrm{MoO}_{4}-$ $A \mathrm{MoO}_{4}-\mathrm{Ga}_{2}\left(\mathrm{MoO}_{4}\right)_{3}$ systems triple molybdates of composition $\mathrm{Ag} A_{3} \mathrm{Ga}\left(\mathrm{MoO}_{4}\right)_{5}$ were found. These compounds were synthesized by the solid-state reactions at 550$600^{\circ} \mathrm{C}(A=\mathrm{Mn}), 540-550^{\circ} \mathrm{C}(A=\mathrm{Zn})$, $500-530^{\circ} \mathrm{C}(A=\mathrm{Co})$ for $80-100 \mathrm{~h}$. However, nickel-containing compound was not obtained in the single-phase state, even after sintering at temperatures as high as 600$650^{\circ} \mathrm{C}$ for $250-300$ hours. This may be due to the smallest radius of $\mathrm{Ni}^{2+}$ cation $(0.69 \AA$ for $\mathrm{CN}=6$ [17]) in the studied series of simple molybdates of divalent metals.

The triple molybdates $\mathrm{Ag} \mathrm{A}_{3} \mathrm{Ga}\left(\mathrm{MoO}_{4}\right)_{5}$ ( $\mathrm{A}=\mathrm{Zn}, \mathrm{Mn}, \mathrm{Co})$ were found to melt incongruently at temperatures of 644,727 , and $739^{\circ} \mathrm{C}$, respectively.

The powder XRD patterns of asprepared single-phase compounds $\mathrm{Ag} A_{3} \mathrm{Ga}\left(\mathrm{MoO}_{4}\right)_{5}$ are similar and show that these oxides are isostructural to triclinic $\mathrm{NaMg}_{3} \mathrm{In}\left(\mathrm{MoO}_{4}\right)_{5}$ (sp. gr. $P \overline{1}, \mathrm{Z}=2$ ) [20]. The diffractograms of the $\mathrm{Ag} A_{3} \mathrm{Ga}\left(\mathrm{MoO}_{4}\right)_{5}$ ( $A=\mathrm{Mn}, \mathrm{Co}, \mathrm{Zn}$ ) were indexed with taking into account our data obtained earlier in the course of single-crystal structure de- 
termination of $\operatorname{AgMg}_{3} R\left(\mathrm{MoO}_{4}\right)_{5}, R=\mathrm{Fe}$, $\mathrm{Cr}$ [7]. The result of indexing the PXRD patterns for $\operatorname{Ag}_{3} \mathrm{Ga}\left(\mathrm{MoO}_{4}\right)_{5}(A=\mathrm{Mn}$, $\mathrm{Co}, \mathrm{Zn})$ are given in Table 1 . Unit-cell parameters are listed in Table 2.

Crystal structure of $\mathrm{AgZn} \mathrm{Ga}_{3}\left(\mathrm{MoO}_{4}\right)_{5}$

The crystal structure of $\mathrm{AgZn} \mathrm{Z}_{3} \mathrm{Ga}\left(\mathrm{MoO}_{4}\right)_{5}$ was refined ac- cording to the Rietveld method [21], starting with the atomic coordinates of $\mathrm{AgMg}_{3} \mathrm{Fe}\left(\mathrm{MoO}_{4}\right)_{5}$ structure [7]. Crystal data, data collection and structure refinement details are summarized in Table 3. Experimental, theoretical, and difference PXRD patterns for the $\mathrm{AgZn} \mathrm{Z}_{3} \mathrm{Ga}\left(\mathrm{MoO}_{4}\right)_{5}$ are shown in Figure 1. The fractional

Table 1

The calculated and observed values of PXRD data for $\operatorname{Ag} A_{3} \mathrm{Ga}\left(\mathrm{MoO}_{4}\right)_{5}(A=\mathrm{Mn}, \mathrm{Zn}, \mathrm{Co})$

\begin{tabular}{|c|c|c|c|c|c|c|c|c|c|c|c|}
\hline \multirow{2}{*}{$\mathrm{h}$} & \multirow{2}{*}{$\mathrm{k}$} & \multirow{2}{*}{1} & \multicolumn{3}{|c|}{$\mathrm{AgMn}_{3} \mathrm{Ga}\left(\mathrm{MoO}_{4}\right)_{5}$} & \multicolumn{3}{|c|}{$\mathrm{AgZn}{ }_{3} \mathrm{Ga}\left(\mathrm{MoO}_{4}\right)_{5}$} & \multicolumn{3}{|c|}{$\mathrm{AgCo}_{3} \mathrm{Ga}\left(\mathrm{MoO}_{4}\right)_{5}$} \\
\hline & & & $\mathrm{I} / \mathrm{I}_{0}$ & $2 \theta_{\text {obs. }}{ }^{\circ}$ & $2 \theta_{\text {cal. }}{ }^{\circ}$ & $\mathrm{I} / \mathrm{I}_{0}$ & $2 \theta_{\text {obs. }}{ }^{\circ}$ & $2 \theta_{\text {cal. }}{ }^{\circ}$ & $\mathrm{I} / \mathrm{I}_{0}$ & $2 \theta_{\text {obs. }}{ }^{\circ}$ & $2 \theta_{\text {cal. }}{ }^{\circ}$ \\
\hline 0 & 0 & 2 & 3 & 9.861 & 9.850 & 2 & 9.981 & 9.991 & 2 & 10.006 & 10.019 \\
\hline 0 & 1 & 0 & 1 & 12.782 & 12.767 & 1 & 12.954 & 12.945 & $1 \mathrm{~L}$ & 12.956 & 12.943 \\
\hline 1 & 0 & 0 & 9 & 12.903 & 12.894 & 9 & 13.058 & 13.064 & 9 & 13.112 & 13.108 \\
\hline 0 & 1 & 1 & & & & & & & $1 \mathrm{~L}$ & 13.769 & 13.750 \\
\hline 1 & 0 & 1 & $1 \mathrm{~L}$ & 13.654 & 13.637 & 1 & 13.778 & 13.808 & $1 \mathrm{~L}$ & 13.853 & 13.849 \\
\hline 0 & -1 & 1 & & & & $1 \mathrm{~L}$ & 14.021 & 13.998 & $1 \mathrm{~L}$ & 14.005 & 14.017 \\
\hline-1 & 0 & 1 & $1 \mathrm{~L}$ & 13.974 & 13.977 & 2 & 14.170 & 14.175 & 1 & 14.231 & 14.226 \\
\hline 0 & 0 & 3 & $1 \mathrm{~L}$ & 14.805 & 14.798 & 1 & 15.010 & 15.011 & $1 \mathrm{~L}$ & 15.061 & 15.052 \\
\hline 1 & 0 & 2 & 1 & 15.924 & 15.959 & $1 \mathrm{~L}$ & 16.154 & 16.157 & 1 & 16.177 & 16.200 \\
\hline 0 & -1 & 2 & $1 \mathrm{~L}$ & 16.388 & 16.393 & $1 \mathrm{~L}$ & 16.577 & 16.576 & 1 & 16.625 & 16.619 \\
\hline-1 & 0 & 2 & $1 \mathrm{~L}$ & 16.529 & 16.538 & $1 \mathrm{~L}$ & 16.799 & 16.783 & $1 \mathrm{~L}$ & 16.819 & 16.844 \\
\hline 1 & 1 & 1 & & & & $1 \mathrm{~L}$ & 17.079 & 17.075 & & & \\
\hline-1 & -1 & 1 & & & & $1 \mathrm{~L}$ & 17.541 & 17.562 & $1 \mathrm{~L}$ & 17.596 & 17.610 \\
\hline 1 & 1 & 2 & $1 \mathrm{~L}$ & 18.728 & 18.728 & & & & & & \\
\hline 1 & 0 & 3 & $1 \mathrm{~L}$ & 19.304 & 19.310 & & & & & & \\
\hline-1 & -1 & 2 & 2 & 19.638 & 19.630 & 3 & 19.826 & 19.822 & 3 & 19.892 & 19.891 \\
\hline 0 & 0 & 4 & \multirow{2}{*}{1} & \multirow{2}{*}{19.780} & 19.774 & & & & \multirow{2}{*}{$1 \mathrm{~L}$} & \multirow{2}{*}{20.127} & 20.115 \\
\hline-1 & 1 & 0 & & & 19.792 & $1 \mathrm{~L}$ & 20.115 & 20.122 & & & 20.154 \\
\hline-1 & 0 & 3 & $1 \mathrm{~L}$ & 20.025 & 20.033 & $1 \mathrm{~L}$ & 20.340 & 20.336 & & & \\
\hline-1 & 1 & 1 & $1 \mathrm{~L}$ & 20.406 & 20.425 & $1 \mathrm{~L}$ & 20.774 & 20.790 & $1 \mathrm{~L}$ & 20.819 & 20.817 \\
\hline 1 & 1 & 3 & 3 & 21.572 & 21.577 & 4 & 21.846 & 21.856 & 5 & 21.861 & 21.864 \\
\hline 1 & -1 & 2 & 6 & 22.117 & 22.117 & 10 & 22.419 & 22.427 & 10 & 22.483 & 22.482 \\
\hline-1 & -1 & 3 & & & & 1 & 22.959 & 22.996 & $1 \mathrm{~L}$ & 23.048 & 23.085 \\
\hline 1 & 0 & 4 & \multirow{2}{*}{3} & \multirow{2}{*}{23.267} & 23.270 & 3 & 23.569 & 23.573 & 3 & 23.646 & 23.631 \\
\hline 0 & 1 & 4 & & & 23.265 & 2 & 23.662 & 23.668 & 1 & 23.695 & 23.675 \\
\hline 0 & -1 & 4 & 1 & 23.959 & 23.944 & 10 & 24.193 & 24.222 & 3 & 24.313 & 24.306 \\
\hline-1 & 0 & 4 & 1 & 24.086 & 24.078 & 2 & 24.441 & 24.445 & 1 & 24.511 & 24.529 \\
\hline 0 & 0 & 5 & 100 & 24.793 & 24.787 & 100 & 25.145 & 25.148 & 86 & 25.221 & 25.218 \\
\hline-1 & 1 & 3 & 6 & 24.869 & 24.848 & 16 & 25.307 & 25.312 & 16 & 25.344 & 25.346 \\
\hline
\end{tabular}


Continuation of table 1

\begin{tabular}{|c|c|c|c|c|c|c|c|c|c|c|c|}
\hline \multirow{2}{*}{$\mathrm{h}$} & \multirow{2}{*}{$\mathrm{k}$} & \multirow{2}{*}{1} & \multicolumn{3}{|c|}{$\mathrm{AgMn}_{3} \mathrm{Ga}\left(\mathrm{MoO}_{4}\right)_{5}$} & \multicolumn{3}{|c|}{$\mathrm{AgZn}_{3} \mathrm{Ga}\left(\mathrm{MoO}_{4}\right)_{5}$} & \multicolumn{3}{|c|}{$\mathrm{AgCo}_{3} \mathrm{Ga}\left(\mathrm{MoO}_{4}\right)_{5}$} \\
\hline & & & $\mathrm{I} / \mathrm{I}_{0}$ & $2 \theta_{\text {obs. }}{ }^{\circ}$ & $2 \theta_{\text {cal. }}{ }^{\circ}$ & $\mathrm{I} / \mathrm{I}_{0}$ & $2 \theta_{\text {obs. }}{ }^{\circ}$ & $2 \theta_{\text {cal. }}{ }^{\circ}$ & $\mathrm{I} / \mathrm{I}_{0}$ & $2 \theta_{\text {obs. }}{ }^{\circ}$ & $2 \theta_{\text {cal. }}{ }^{\circ}$ \\
\hline 1 & 1 & 4 & 3 & 25.122 & 25.119 & 5 & 25.459 & 25.465 & 6 & 25.480 & 25.478 \\
\hline 0 & 2 & & 3 & 5.701 & 25.697 & 2 & 26.072 & 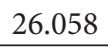 & 2 & 055 & 6.054 \\
\hline 2 & 0 & & 73 & 5.959 & 29.954 & 83 & 26.307 & 26.302 & \multirow{2}{*}{100} & \multirow{2}{*}{26.396} & 26.392 \\
\hline 0 & 2 & 1 & 23 & 26.036 & 26.025 & 38 & 26.417 & 26.422 & & & 26.403 \\
\hline 2 & 0 & 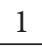 & 10 & 26.255 & 26.250 & 20 & 26.596 & 26.590 & 18 & 6.679 & 26.676 \\
\hline 0 & -2 & 1 & 6 & 6.337 & 26.333 & 22 & 26.664 & 26.674 & 7 & 97 & 26.690 \\
\hline-1 & -1 & 1 & 4 & 83 & 26.488 & 7 & 26.780 & 26.782 & \multirow{2}{*}{7} & \multirow{2}{*}{26.888} & 26.890 \\
\hline 1 & 2 & 0 & \multirow{2}{*}{8} & \multirow{2}{*}{26.613} & 26.601 & 3 & 26.883 & 26.881 & & & 26.892 \\
\hline-2 & 0 & 1 & & & 26.614 & 7 & 26.984 & 26.984 & 7 & 27.079 & 27.081 \\
\hline 1 & 2 & 1 & 11 & 26.835 & 26.829 & 25 & 27.132 & 27.137 & 18 & 27.133 & 27.131 \\
\hline 2 & 1 & 1 & 2 & 7.007 & 27.001 & 5 & 27.283 & 27.278 & 3 & 27.350 & 27.345 \\
\hline-1 & -2 & 1 & \multirow{2}{*}{11} & \multirow{2}{*}{27.307} & 27.306 & 26 & 27.575 & 27.575 & 19 & 27.613 & 27.609 \\
\hline 0 & 2 & 2 & & & 27.285 & 4 & 27.712 & 27.731 & 3 & 27.705 & 27.703 \\
\hline-2 & -1 & 1 & 3 & 27.507 & 27.503 & 4 & 27.790 & 27.784 & 3 & 27.880 & 27.879 \\
\hline 0 & 1 & 5 & 6 & 27.622 & 27.611 & 10 & 28.081 & 28.084 & 8 & 28.109 & 28.105 \\
\hline 0 & -2 & 2 & 3 & 27.874 & 27.873 & 6 & 28.208 & 28.210 & 5 & 28.255 & 28.250 \\
\hline 1 & 2 & 2 & 2 & 27.961 & 27.970 & 4 & 28.320 & 28.322 & 4 & 28.308 & 28.304 \\
\hline 1 & -1 & 4 & 2 & 28.066 & 28.064 & 6 & 28.423 & 28.425 & 5 & 28.512 & 28.510 \\
\hline 2 & 1 & 2 & 2 & 28.114 & 28.121 & & & 28.423 & 3 & 28.454 & 28.481 \\
\hline-2 & 0 & 2 & 2 & 28.164 & 28.167 & 1 & 28.567 & 28.573 & 2 & 28.672 & 28.678 \\
\hline 0 & -1 & 5 & 6 & 28.342 & 28.336 & 16 & 28.678 & 28.676 & 13 & 28.782 & 28.778 \\
\hline-1 & 0 & 5 & & & & 1 & 28.893 & 28.900 & 1 & 29.000 & 28.997 \\
\hline-1 & -2 & 2 & 1 & 28.877 & 28.882 & 2 & 29.163 & 29.159 & 1 & 29.223 & 29.217 \\
\hline-2 & -1 & 2 & 4 & 29.081 & 29.082 & 10 & 29.391 & 29.390 & 9 & 29.499 & 29.501 \\
\hline 0 & 2 & 3 & 3 & 9.376 & 29.367 & 4 & 29.859 & 29.869 & 3 & 9.837 & 29.839 \\
\hline 2 & 0 & 3 & $1 \mathrm{~L}$ & 29.525 & 29.516 & 2 & 29.898 & 29.888 & 1 & 29.985 & 29.973 \\
\hline 0 & 0 & 6 & 1 & 29.860 & 29.850 & 1 & 30.300 & 30.287 & 1 & 30.383 & 30.372 \\
\hline 1 & 2 & 3 & $1 \mathrm{~L}$ & 29.929 & 29.927 & 1 & 30.365 & 30.334 & 1 & 30.324 & 30.310 \\
\hline 2 & 1 & 3 & 4 & & 30.056 & 9 & 30.397 & 397 & 9 & & 30.450 \\
\hline 0 & -2 & 3 & $1 \mathrm{~L}$ & & 30.189 & 1 & & 30.539 & 1 & 30.590 & 30.603 \\
\hline-2 & 0 & 3 & $1 \mathrm{~L}$ & 30.513 & 30.488 & 1 & & 30.940 & $1 \mathrm{~L}$ & 31.055 & 31.055 \\
\hline$-1-1$ & & & $1 \mathrm{~L}$ & 30.622 & 30.621 & 1 & & 30.981 & 1 & 31.088 & 31.106 \\
\hline-1 & 2 & 0 & 1 & 30.953 & 30.949 & 2 & & 31.462 & 1 & 483 & 31.484 \\
\hline-1 & -2 & 3 & 2 & 31.213 & 31.207 & 4 & 31.501 & 31.509 & 4 & 31.594 & 31.592 \\
\hline-2 & 1 & 0 & & & & 1 & & & $1 \mathrm{~L}$ & & 31.698 \\
\hline-2 & -1 & 3 & & & & 2 & 31.748 & 31.755 & 18 & & 31.883 \\
\hline-1 & 2 & 1 & 8 & 31.303 & 1.304 & 22 & 31.849 & 31.854 & 10 & 005 & 31.865 \\
\hline
\end{tabular}


Continuation of table 1

\begin{tabular}{|c|c|c|c|c|c|c|c|c|c|c|c|}
\hline \multirow{2}{*}{$\mathrm{h}$} & \multirow{2}{*}{$\mathrm{k}$} & \multirow{2}{*}{1} & \multicolumn{3}{|c|}{$\mathrm{AgMn}_{3} \mathrm{Ga}\left(\mathrm{MoO}_{4}\right)_{5}$} & \multicolumn{3}{|c|}{$\mathrm{AgZn}_{3} \mathrm{Ga}\left(\mathrm{MoO}_{4}\right)_{5}$} & \multicolumn{3}{|c|}{$\mathrm{AgCo}_{3} \mathrm{Ga}\left(\mathrm{MoO}_{4}\right)_{5}$} \\
\hline & & & $\mathrm{I} / \mathrm{I}_{0}$ & $2 \theta_{\text {obs. }}{ }^{\circ}$ & $2 \theta_{\text {cal. }}^{\circ}$ & $\mathrm{I} / \mathrm{I}_{0}$ & $2 \theta_{\text {obs. }}{ }^{\circ}$ & $2 \theta_{\text {cal. }}{ }^{\circ}$ & $\mathrm{I} / \mathrm{I}_{0}$ & $2 \theta_{\text {obs. }}{ }^{\circ}$ & $2 \theta_{\text {cal. }}{ }^{\circ}$ \\
\hline 1 & -2 & 1 & 2 & 31.408 & 31.410 & \multirow{2}{*}{2} & \multirow{2}{*}{31.912} & 31.899 & 3 & 31.945 & 31.935 \\
\hline 2 & -1 & 1 & 1 & 31.440 & 31.428 & & & 31.914 & 1 & 31.996 & 31.999 \\
\hline 1 & -1 & 5 & 1 & 31.853 & 31.857 & 3 & 32.264 & 32.264 & 2 & 32.362 & 32.364 \\
\hline-1 & 1 & 5 & $1 \mathrm{~L}$ & 31.974 & 31.980 & & & & 1 & 32.590 & 32.619 \\
\hline 0 & 2 & 4 & 2 & 32.137 & 32.125 & \multirow{2}{*}{9} & \multirow{2}{*}{32.653} & 32.689 & 2 & 32.654 & 32.663 \\
\hline 2 & 0 & 4 & 4 & 32.239 & 32.238 & & & 32.647 & 6 & 32.741 & 32.736 \\
\hline 2 & -1 & 2 & 1 & 32.512 & 32.535 & \multirow{2}{*}{11} & \multirow{2}{*}{33.027} & 33.015 & 1 & 33.127 & 33.106 \\
\hline 1 & 2 & 4 & 3 & 32.569 & 32.567 & & & 33.038 & 7 & 33.020 & 33.014 \\
\hline 1 & -2 & 2 & 3 & 32.655 & 32.656 & 5 & 33.131 & 33.133 & 4 & 33 & 33.186 \\
\hline 0 & -1 & 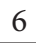 & & & & 2 & 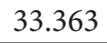 & 33.368 & & & \\
\hline 0 & -2 & 4 & 7 & 80.0 & 34 & \multirow{2}{*}{16} & \multirow{2}{*}{33.511} & 33.513 & 11 & 33.603 & 33.601 \\
\hline 2 & 2 & 0 & 1 & 33.214 & 33.200 & & & 33.510 & 1 & 33.551 & 33.568 \\
\hline 2 & 2 & 1 & 2 & 33.311 & 33.313 & 6 & 33.634 & 33.641 & 5 & 33.683 & 33.681 \\
\hline-2 & 0 & 4 & 1 & 33.426 & 33.434 & \multirow{2}{*}{4} & \multirow{2}{*}{33.935} & 33.941 & 1 & 34.059 & 34.066 \\
\hline 1 & 1 & 6 & $1 \mathrm{~L}$ & 33.460 & 33.454 & & & 33.948 & & & \\
\hline-2 & -2 & 1 & 3 & 33.847 & 33.852 & 11 & 34.158 & 34.160 & 8 & 34.239 & 34.239 \\
\hline
\end{tabular}

$\mathrm{Cu}$ Ka1 radiation $(l=1.54056 \AA)$

Table 2

Unit-cell parameters for $\mathrm{Ag} A_{3} \mathrm{Ga}\left(\mathrm{MoO}_{4}\right)_{5}(A=\mathrm{Mn}, \mathrm{Zn}, \mathrm{Co})$

\begin{tabular}{c|c|c|c|c|c|c|c}
\hline$A$ & $a, \AA$ & $b, \AA$ & $c, \AA$ & $\alpha^{\circ}$ & $\beta^{\circ}$ & $\gamma^{\circ}$ & $V, \AA^{3}$ \\
\hline $\mathrm{Mn}$ & $6.9844(3)$ & $7.0519(4)$ & $17.9700(8)$ & $87.796(4)$ & $87.529(5)$ & $79.386(4)$ & 868.71 \\
\hline $\mathrm{Zn}$ & $6.9037(3)$ & $6.9639(4)$ & $17.7147(8)$ & $88.107(4)$ & $87.440(4)$ & $78.982(4)$ & 834.87 \\
\hline $\mathrm{Co}$ & $6.8810(4)$ & $6.9657(4)$ & $17.669(1)$ & $87.895(5)$ & $87.344(5)$ & $78.976(5)$ & 830.04
\end{tabular}

atomic coordinates, isotropic atomic displacement parameters, cation occupancies and main selected interatomic distances are presented in Tables 4 and 5. The populations of four independent positions $M=(\mathrm{Zn}, \mathrm{Ga})$ and three incompletely occupied Ag sites were refined with keeping the electrical neutrality of the chemical formula. The final compositions of the crystals are close to stoichiometric $\mathrm{AgZn}{ }_{3} \mathrm{Ga}\left(\mathrm{MoO}_{4}\right)_{5}$ with a negligible silver deficiency.

In the structure $\left.\left.\mathrm{AgZn} \mathrm{Zna}_{3} \mathrm{GaO}\right)_{5}\right)_{5}$ all atoms are located in general positions. Coordination polyhedra of Mo atoms are tetrahedra with Mo-O distances of 1.714$1.824 \AA$, which are similar to the values found in other molybdates containing a tetrahedral anion. Cations $\mathrm{Zn}^{2+}$ and $\mathrm{Ga}^{3+}$ are statistically distributed on octahedral positions $M 1-M 4$ with the $(\mathrm{Zn}, \mathrm{Ga})-\mathrm{O}$ bond lengths of 1.940-2.129 $\AA$. Both Ag1 and Ag3 cations are coordinated by four $\mathrm{O}$ atoms (Ag1-O 2.358 A, Ag3-O 2.415 ̊), while Ag2 cation has $\mathrm{CN}=5$ (Ag2-O $2.495 \AA$ ). The structure of $\mathrm{AgZn}_{3} \mathrm{Ga}\left(\mathrm{MoO}_{4}\right)_{5}$ consists of $\mathrm{MoO}_{4}$ tetrahedra, couples of edgeshared $M(1) \mathrm{O}_{6}$ octahedra, and trimers of edge-shared $M(2) \mathrm{O}_{6}, M(3) \mathrm{O}_{6}$ and 
Crystal data and structure refinement for $\mathrm{AgZn}_{3} \mathrm{Ga}\left(\mathrm{MoO}_{4}\right)_{5}$

\begin{tabular}{c|c}
\hline Structural formula & $\mathrm{AgZn}_{3} \mathrm{Ga}\left(\mathrm{MoO}_{4}\right)_{5}$ \\
\hline Formula weight, $M_{r}\left(\mathrm{~g} \mathrm{~mol}^{-1}\right)$ & 1172.58 \\
\hline Temperature $(\mathrm{K})$ & $298(2)$ \\
\hline Crystal system, space group $(\#)$ & Triclinic, $P \overline{1}(2)$ \\
\hline Unit-cell parameters: & \\
$a(\AA)$ & $6.9035(5)$ \\
$b(\AA)$ & $6.9643(5)$ \\
$c(\AA)$ & $17.7160(14)$ \\
$\alpha\left(^{\circ}\right)$ & $88.1039(11)$ \\
$\beta\left(^{\circ}\right)$ & $87.4338(12)$ \\
$\gamma\left(^{\circ}\right)$ & $78.9880(9)$ \\
\hline Unit-cell volume, $V\left(\AA^{3}\right)$ & $835.0(2)$ \\
\hline Formula unit, $Z$ & 2 \\
\hline Calculated density, $\rho_{\text {cal }}\left(\mathrm{g} \mathrm{cm}^{-3}\right)$ & 4.66 \\
\hline Refinement $R$ factors and goodness of fit: & \\
$w R_{p}$ & 0.0511 \\
$R_{p}$ & 0.0382 \\
$R_{\text {exp }}$ & 0.0152 \\
$R\left(F^{2}\right)$ & 0.05815 \\
$\chi^{2}$ & 3.40
\end{tabular}

Table 4

Structural parameters for $\mathrm{AgZn} \mathrm{Ga}_{3}\left(\mathrm{MoO}_{4}\right)_{5}$

\begin{tabular}{c|c|c|c|c|c}
\hline Atom & Occupancy & $\boldsymbol{x}$ & $\boldsymbol{y}$ & $\boldsymbol{z}$ & Uiso \\
\hline Mo1 & 1 & $0.2722(8)$ & $0.3095(8)$ & $0.5282(3)$ & $0.030(2)$ \\
\hline Mo2 & 1 & $0.2129(8)$ & $0.8293(9)$ & $0.2856(3)$ & $0.028(2)$ \\
\hline Mo3 & 1 & $0.6843(8)$ & $0.2187(8)$ & $0.3109(3)$ & $0.023(2)$ \\
\hline Mo4 & 1 & $0.2811(9)$ & $0.0522(9)$ & $0.9044(3)$ & $0.029(2)$ \\
\hline Mo5 & 1 & $0.2520(8)$ & $0.5491(8)$ & $0.0863(3)$ & $0.021(2)$ \\
\hline M1 & $0.788(1) \mathrm{Zn}+0.212(1) \mathrm{Ga}$ & $0.1834(12)$ & $0.8241(11)$ & $0.4938(5)$ & $0.0126(3)$ \\
\hline M2 & $0.901(1) \mathrm{Zn}+0.099(1) \mathrm{Ga}$ & $0.1704(14)$ & $0.0855(16)$ & $0.1145(5)$ & $0.045(4)$ \\
\hline M3 & $0.798(1) \mathrm{Zn}+0.202(1) \mathrm{Ga}$ & $0.7829(12)$ & $0.4310(13)$ & $0.1239(4)$ & $0.014(3)$ \\
\hline M4 & $0.505(1) \mathrm{Zn}+0.495(1) \mathrm{Ga}$ & $0.2546(12)$ & $0.3014(13)$ & $0.7370(4)$ & $0.023(3)$ \\
\hline Ag1 & $0.323(3) \mathrm{Ag}$ & $0.149(3)$ & $0.339(3)$ & $0.2857(12)$ & $0.062(5)$ \\
\hline Ag2 & $0.328(3) \mathrm{Ag}$ & $0.122(4)$ & $0.308(4)$ & $0.3155(13)$ & $0.062(5)$ \\
\hline Ag3 & $0.342(3) \mathrm{Ag}$ & $0.097(3)$ & $0.370(3)$ & $0.3445(11)$ & $0.062(5)$ \\
\hline O1 & 1 & $0.511(5)$ & $0.194(5)$ & $0.5163(18)$ & $0.015(1)$ \\
\hline O2 & 1 & $0.289(4)$ & $0.366(4)$ & $0.6238(17)$ & $0.015(1)$ \\
\hline O3 & 1 & $0.171(4)$ & $0.545(5)$ & $0.4601(18)$ & $0.015(1)$ \\
\hline O4 & 1 & $0.130(5)$ & $0.126(5)$ & $0.4978(18)$ & $0.015(1)$ \\
\hline & & & &
\end{tabular}


Continuation of table 4

\begin{tabular}{c|c|c|c|c|c}
\hline Atom & Occupancy & $\boldsymbol{x}$ & $\boldsymbol{y}$ & $\boldsymbol{z}$ & Uiso \\
\hline O5 & 1 & $0.189(4)$ & $0.872(4)$ & $0.3866(18)$ & $0.015(1)$ \\
\hline O6 & 1 & $0.477(5)$ & $0.719(4)$ & $0.2580(17)$ & $0.015(1)$ \\
\hline O7 & 1 & $0.140(5)$ & $0.053(5)$ & $0.2220(19)$ & $0.015(1)$ \\
\hline O8 & 1 & $0.098(5)$ & $0.641(5)$ & $0.2687(18)$ & $0.015(1)$ \\
\hline O9 & 1 & $0.419(5)$ & $0.280(4)$ & $0.3590(17)$ & $0.015(1)$ \\
\hline O10 & 1 & $0.804(5)$ & $0.191(4)$ & $0.3830(18)$ & $0.015(1)$ \\
\hline O11 & 1 & $0.681(5)$ & $0.995(5)$ & $0.2696(17)$ & $0.015(1)$ \\
\hline O12 & 1 & $0.774(4)$ & $0.370(5)$ & $0.237(2)$ & $0.015(1)$ \\
\hline O13 & 1 & $0.198(4)$ & $0.121(4)$ & $0.997(2)$ & $0.015(1)$ \\
\hline O14 & 1 & $0.468(5)$ & $0.040(4)$ & $0.0841(15)$ & $0.015(1)$ \\
\hline O15 & 1 & $0.831(5)$ & $0.202(5)$ & $0.1174(16)$ & $0.015(1)$ \\
\hline O16 & 1 & $0.238(4)$ & $0.305(5)$ & $0.8544(17)$ & $0.015(1)$ \\
\hline O17 & 1 & $0.249(4)$ & $0.546(4)$ & $0.987(2)$ & $0.015(1)$ \\
\hline O18 & 1 & $0.485(5)$ & $0.488(5)$ & $0.1153(17)$ & $0.015(1)$ \\
\hline O19 & 1 & $0.171(4)$ & $0.778(5)$ & $0.1292(18)$ & $0.015(1)$ \\
\hline O20 & & $0.097(4)$ & $0.410(5)$ & $0.1173(18)$ & $0.015(1)$
\end{tabular}

$M(4) \mathrm{O}_{6}$ octahedra, which are linked by the common vertices to form a 3D framework (Fig. 2). In the large framework cavities, the silver cations are disordered on three close positions with the distances $\mathrm{Ag}-\mathrm{Ag}$ 0.595(4) A and 1.101(2) ̊.

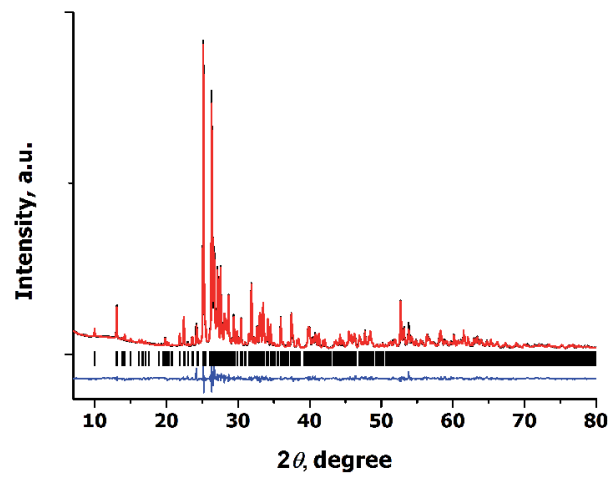

Fig. 1. Observed (black line) and calculated (red line) XRD patterns of $\mathrm{AgZn} \mathrm{Ga}_{3}\left(\mathrm{MoO}_{4}\right)_{5}$. Vertical bars indicate the positions of the Bragg peaks. The lower trace depicts the difference between the experimental and calculated intensity values
Such a disordering is also typical of other compounds of this isostructural series [7, 9], suggesting a possible mobility of the $\mathrm{Ag}^{+}$cations in the compounds. This is favored not only by defects in Ag positions along with their irregular coordina-

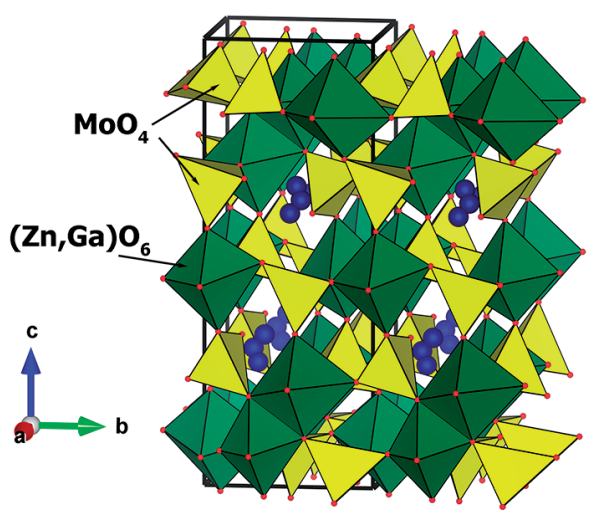

Fig. 2. Projection views of the structure of $\mathrm{AgZn}_{3} \mathrm{Ga}\left(\mathrm{MoO}_{4}\right)_{5}$ along the $a$ axis. The blue spheres and small red spheres indicate $\mathrm{Ag}$ and oxygen atoms, respectively 
Table 5

Selected interatomic distances $(\AA)$ in $\mathrm{AgZn}_{3} \mathrm{Ga}\left(\mathrm{MoO}_{4}\right)_{5}$

\begin{tabular}{|c|c|c|c|}
\hline \multicolumn{2}{|c|}{ Mo1-tetrahedron } & \multicolumn{2}{|c|}{ Mo2-tetrahedron } \\
\hline $\begin{array}{r}\mathrm{Mo1}-\mathrm{O} 1 \\
-\mathrm{O} 2 \\
-\mathrm{O} 3 \\
-\mathrm{O} 4 \\
<\mathrm{Mo} 1-\mathrm{O}>\end{array}$ & $\begin{array}{c}1.696(3) \\
1.764(3) \\
1.726(3) \\
1.858(3) \\
1.761\end{array}$ & $\begin{array}{r}\mathrm{Mo} 2-\mathrm{O} 5 \\
-\mathrm{O} 6 \\
-\mathrm{O} 7 \\
-\mathrm{O} 8 \\
<\mathrm{Mo} 2-\mathrm{O}>\end{array}$ & $\begin{array}{c}1.819(3) \\
1.889(3) \\
1.891(3) \\
1.698(3) \\
1.824\end{array}$ \\
\hline \multicolumn{2}{|c|}{$\mathrm{Mo}^{3}$-tetrahedron } & \multicolumn{2}{|c|}{ Mo4-tetrahedron } \\
\hline $\begin{array}{r}\text { Mo3-O9 } \\
-\mathrm{O} 10 \\
-\mathrm{O} 11 \\
-\mathrm{O} 12 \\
<\mathrm{Mo} 3-\mathrm{O}>\end{array}$ & $\begin{array}{c}1.961(3) \\
1.739(3) \\
1.747(4) \\
1.812(3) \\
1.815\end{array}$ & $\begin{array}{r}\mathrm{Mo} 4-\mathrm{O} 13 \\
-\mathrm{O} 14 \\
-\mathrm{O} 15 \\
-\mathrm{O} 16 \\
<\mathrm{Mo} 4-\mathrm{O}>\end{array}$ & $\begin{array}{c}1.749(3) \\
1.726(2) \\
1.922(3) \\
1.760(3) \\
1.789\end{array}$ \\
\hline \multicolumn{2}{|c|}{ Mo5-tetrahedron } & \multicolumn{2}{|c|}{ M1-octahedron } \\
\hline $\begin{array}{r}\text { Mo5-O17 } \\
-\mathrm{O} 18 \\
-\mathrm{O} 19 \\
-\mathrm{O} 20 \\
<\mathrm{Mo} 5-\mathrm{O}>\end{array}$ & $\begin{array}{c}1.758(4) \\
1.683(3) \\
1.773(3) \\
1.640(3) \\
1.714\end{array}$ & $\begin{array}{c}M 1-\mathrm{O} 1 \\
-\mathrm{O} 3 \\
-\mathrm{O} 10 \\
-\mathrm{O} 4 \\
-\mathrm{O} 5 \\
-\mathrm{O} 4 \\
<M 1-\mathrm{O}>\end{array}$ & $\begin{array}{c}2.092(3) \\
2.068(3) \\
2.186(3) \\
2.070(2) \\
1.918(3) \\
2.124(3) \\
2.076\end{array}$ \\
\hline \multicolumn{2}{|c|}{ M2-octahedron } & \multicolumn{2}{|c|}{ M3-octahedron } \\
\hline $\begin{array}{r}M 2-\mathrm{O} 7 \\
-\mathrm{O} 13 \\
-\mathrm{O} 19 \\
-\mathrm{O} 20 \\
-\mathrm{O} 14 \\
-\mathrm{O} 15 \\
<M 2-\mathrm{O}>\end{array}$ & $\begin{array}{c}1.918(3) \\
2.096(3) \\
2.144(3) \\
2.224(3) \\
2.066(3) \\
2.325(3) \\
2.129\end{array}$ & $\begin{array}{r}M 3-\mathrm{O} 18 \\
-\mathrm{O} 17 \\
-\mathrm{O} 15 \\
-\mathrm{O} 16 \\
-\mathrm{O} 12 \\
-\mathrm{O} 20 \\
<M 3-\mathrm{O}>\end{array}$ & $\begin{array}{c}2.030(3) \\
1.99(4) \\
1.572(3) \\
1.869(3) \\
2.038(3) \\
2.143(3) \\
1.940\end{array}$ \\
\hline \multicolumn{2}{|c|}{ M4-octahedron } & \multicolumn{2}{|c|}{ Ag1-polyhedron } \\
\hline $\begin{array}{c}M 4-\mathrm{O} 8 \\
-\mathrm{O} 11 \\
-\mathrm{O} 2 \\
-\mathrm{O} 6 \\
-\mathrm{O} 16 \\
-\mathrm{O} 12 \\
<M 4-\mathrm{O}>\end{array}$ & $\begin{array}{c}2.398(4) \\
2.033(4) \\
2.053(3) \\
1.837(3) \\
2.079(3) \\
2.320(3) \\
2.120\end{array}$ & $\begin{array}{r}\text { Ag1-O9 } \\
-\mathrm{O} 8 \\
-\mathrm{O} 7 \\
-\mathrm{O} 12 \\
<\mathrm{Ag} 1-\mathrm{O}>\end{array}$ & $\begin{array}{c}2.29(4) \\
2.080(3) \\
2.33(4) \\
2.73(4) \\
2.358\end{array}$ \\
\hline \multicolumn{2}{|c|}{ Ag2-polyhedron } & \multicolumn{2}{|c|}{ Ag3-polyhedron } \\
\hline $\begin{array}{c}\text { Ag2-O9 } \\
-\mathrm{O} 10 \\
-\mathrm{O} 8 \\
-\mathrm{O} 7 \\
-\mathrm{O} 12 \\
<\mathrm{Ag} 2-\mathrm{O}>\end{array}$ & $\begin{array}{c}2.19(4) \\
2.70(4) \\
2.41(4) \\
2.45(4) \\
2.725(3) \\
2.495\end{array}$ & $\begin{array}{r}\mathrm{Ag} 3-\mathrm{O} 9 \\
-\mathrm{O} 8 \\
-\mathrm{O} 10 \\
-\mathrm{O} 3 \\
<\mathrm{Ag} 3-\mathrm{O}>\end{array}$ & $\begin{array}{c}2.22(4) \\
2.28(4) \\
2.62(4) \\
2.540(3) \\
2.415\end{array}$ \\
\hline $\begin{array}{l}\text { Ag1-Ag2 } \\
\text { Ag1-Ag3 }\end{array}$ & $\begin{array}{l}0.595(4) \\
1.101(2)\end{array}$ & & \\
\hline
\end{tabular}


tion, but also a rather flexible polyhedral framework of the $\mathrm{NaMg}_{3} \mathrm{In}\left(\mathrm{MoO}_{4}\right)_{5}$ structure type, which involves interconnected cavities.

\section{Electrophysical properties}

As was noted in the previous section, the structural features of the obtained molybdates allow us to expect these compounds to have the increased ionic conductivity. This was already confirmed by us in the case of $\mathrm{AgMg}_{3} \mathrm{Al}\left(\mathrm{MoO}_{4}\right)_{5}(\sigma=$ $\left.=2.5 \times 10^{-2} \mathrm{~S} / \mathrm{cm}\right)$ and $\mathrm{AgMn}_{3} \mathrm{Al}\left(\mathrm{MoO}_{4}\right)_{5}$ $\left(\sigma=7.1 \times 10^{-3} \mathrm{~S} / \mathrm{cm}\right)$ at $500^{\circ} \mathrm{C}$ [7]. In this work as an example, the results of studying electrophysical properties for $\mathrm{AgMn}_{3} \mathrm{Ga}$ $\left(\mathrm{MoO}_{4}\right)_{5}$ are presented.

It was found that the $\mathrm{DC}$ conductivity of ceramic sample $\mathrm{AgMn}_{3} \mathrm{Ga}\left(\mathrm{MoO}_{4}\right)_{5}$, measured with the V7-38 device, is negligible as compared to the ac conductivity (Fig. 3) in temperature region of $100-560^{\circ} \mathrm{C}$. As the platinum electrodes are blocking in the DC conductivity measurement mode, the DC conductivity of $\mathrm{AgMn}_{3} \mathrm{Ga}\left(\mathrm{MoO}_{4}\right)_{5}$ corresponds to the electronic one. Therefore, it can be concluded that the AC conductivity is almost equal to the ionic one.

It is seen that near room temperature the conductivity is as small as $10^{-7} \mathrm{~S} / \mathrm{cm}$

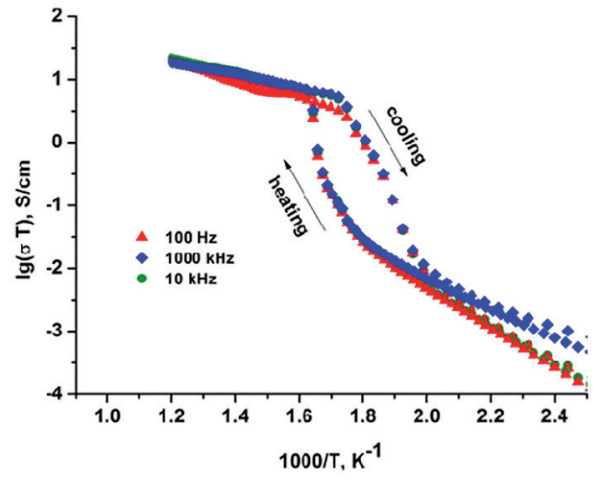

Fig. 3. Temperature dependences of the ionic conductivity on heating and cooling for $\mathrm{AgMn}_{3} \mathrm{Ga}\left(\mathrm{MoO}_{4}\right)_{5}$

but quickly rises with temperature to values of about $10^{-2} \mathrm{~S} / \mathrm{cm}$. It is noteworthy that the conductivity in $\mathrm{AgMn}_{3} \mathrm{Ga}\left(\mathrm{MoO}_{4}\right)_{5}$ increases with temperature in non-monotonic way showing distinct breaks on $\lg \sigma=\mathrm{f}(1 / T)$ curves at $310^{\circ} \mathrm{C}$. Above these temperature the $\lg \sigma=\mathrm{f}(1 / T)$ dependences are almost linear with the small activation energy value $E_{a}=0.26 \mathrm{eV}$. Above $310^{\circ} \mathrm{C}$, the ionic conductivity of $\mathrm{AgMn}_{3} \mathrm{Ga}\left(\mathrm{MoO}_{4}\right)_{5}$ increases up to $2.03 \cdot 10^{-2} \mathrm{~S} / \mathrm{cm}$ at $500^{\circ} \mathrm{C}$, which is close to the corresponding characteristics of the known ionic conductors.

\section{Conclusions}

The possibility of the formation of silver-containing gallium triple molybdates with $\mathrm{Mn}, \mathrm{Co}, \mathrm{Zn}, \mathrm{Ni}$, analogous to the phases $\mathrm{Ag}_{1-x} A_{1-x} R_{1+x}\left(\mathrm{MoO}_{4}\right)_{3}$ and $\operatorname{Ag} A_{3} R\left(\mathrm{MoO}_{4}\right)_{5}$ obtained by us in the $\mathrm{Ag}_{2} \mathrm{MoO}_{4}-\mathrm{AMoO}_{4}-R_{2}\left(\mathrm{MoO}_{4}\right)_{3}(A=\mathrm{Mg}$, Co; $R=\mathrm{Al} ; A=\mathrm{Mg}, R=\mathrm{In}$ ) systems, was studied. It was shown that in the $\mathrm{Ag}_{2} \mathrm{MoO}_{4}-$ $A \mathrm{MoO}_{4}-\mathrm{Ga}_{2}\left(\mathrm{MoO}_{4}\right)_{3}(A=\mathrm{Mn}, \mathrm{Co}, \mathrm{Zn}, \mathrm{Ni})$ systems the NASICON-like phases of the composition $\mathrm{Ag}_{1-x} A_{1-x} R_{1+x}\left(\mathrm{MoO}_{4}\right)_{3}$ are not formed. The triple molybdates of the composition $\mathrm{Ag} A_{3} \mathrm{Ga}\left(\mathrm{MoO}_{4}\right)_{5}(A=\mathrm{Mn}$,
Co, $\mathrm{Zn}$ ) were synthesized and characterized. $\mathrm{AgNi}_{3} \mathrm{Ga}\left(\mathrm{MoO}_{4}\right)_{5}$ was not obtained in the single-phase state. It was established that the obtained compounds incongruently melt and belong to the structural type of triclinic $\mathrm{NaMg}_{3} \mathrm{In}\left(\mathrm{MoO}_{4}\right)_{5}$ (sp. gr. $P \overline{1}, \mathrm{Z}=2)$. The structure of the obtained compounds was refined by the Rietveld method using the powder diffraction data for $\mathrm{AgZn}_{3} \mathrm{Ga}\left(\mathrm{MoO}_{4}\right)_{5}$. The structural features of the obtained molybdates allow us to expect these compounds to have the increased ionic conductivity. This was 
confirmed by studying electrophysical properties of $\mathrm{AgMn}_{3} \mathrm{Ga}\left(\mathrm{MoO}_{4}\right)_{5}$. It was shown that the high-temperature electrical conductivity of this compound reaches
$10^{-2} \mathrm{~S} / \mathrm{cm}$ at $E_{a}=0.26 \mathrm{eV}$, which is close to the corresponding characteristics of the known ionic conductors.

\section{Acknowledgments}

The research was carried out within the state assignment of BINM SB RAS, supported in part by the Russian Foundation for Basic Research (Project No. 17-03-00333a)

\section{References}

1. Kotova IYu. Phase formation in the $\mathrm{Ag}_{2} \mathrm{MoO}_{4}-\mathrm{CoMoO}_{4}-\mathrm{Al}_{2}\left(\mathrm{MoO}_{4}\right)_{3}$ system. Russ J Inorg Chem. 2014;59:844-8. DOI:10.7868/S0044457X14080133.

2. Kotova IYu, Korsun VP. Phase in the $\mathrm{Ag}_{2} \mathrm{MoO}_{4}-\mathrm{MgMoO}_{4}-\mathrm{Al}_{2}\left(\mathrm{MoO}_{4}\right)_{3}$. Russ J Inorg Chem. 2010;55:955-8. DOI: 10.1134/S0036023610060203.

3. Kotova IYu, Korsun VP. Phase formation in the system involving silver, magnesium, and indium molybdates. Russ J Inorg Chem. 2010;55:1965-9. DOI: $10.1134 /$ S0036023610120247.

4. Kotova IYu, Belov DA, Stefanovich SYu. $\mathrm{Ag}_{1-x} \mathrm{Mg}_{1-x} \mathrm{R}_{1+x}\left(\mathrm{MoO}_{4}\right)_{3} \mathrm{Ag}^{+}$-conducting NASICON-like phases, where $\mathrm{R}=\mathrm{Al}$ or Sc and $0 \leq x \leq 0.5$. Russ J Inorg Chem. 2011;56:1189-93. DOI: 10.1134/S0036023611080122.

5. Bouzidi C, Frigui W, Zid MF. Synthèse et structure crystalline d'un matériau noir $\mathrm{AgMn}_{3}{ }_{3}^{\mathrm{II}}\left(\mathrm{Mn}_{0.26}^{\mathrm{III}} \mathrm{Al}_{0.74}\right)\left(\mathrm{MoO}_{4}\right)_{5}$. Acta Cryst. 2015; E71:299-304. DOI: $10.1107 /$ S2056989015003345.

6. Nasri R, Chérif SF, Zid MF. Structure cristalline de la triple molybdate $\mathrm{Ag}_{0.90} \mathrm{Al}_{1.06} \mathrm{Co}_{2.94}$ $\left(\mathrm{MoO}_{4}\right)_{5}$. Acta Cryst. 2015; E71:388-91. DOI: 10.1107/s2056989015005290.

7. Kotova IYu, Solodovnikov SF, Solodovnikova ZA, Belov DA, Stefanovich SYu, Savina AA, Khaikina EG. New series of triple molybdates $\mathrm{Ag}_{3} R\left(\mathrm{MoO}_{4}\right)_{5}(A=\mathrm{Mg}$, $\mathrm{R}=\mathrm{Cr}, \mathrm{Fe} ; A=\mathrm{Mn}, R=\mathrm{Al}, \mathrm{Cr}, \mathrm{Fe}, \mathrm{Sc}, \mathrm{In}$ ) with framework structures and mobile silver ion sublattices. J Solid State Chem. 2016;238:121-8. DOI: 10.1016/j. jssc. 2016.03.003.

8. Balsanova LV. Synthesis of crystals of silver containing oxide phases based on molybdenum, study of their structure and properties. Vestnik VSGUTU. 2015;5:63-9.

9. Kotova IYu, Savina AA, Khaikina EG. Crystal structure of new triple molybdate $\mathrm{AgMg}_{3} \mathrm{Ga}\left(\mathrm{MoO}_{4}\right)_{5}$ from Rietveld refinement. Powder Diffraction. 2017;32(4):255-60. DOI: $10.1017 /$ S0885715617000811.

10. Yanushkevich TM, Zhukovsky VM, Ustyantsev VM. Fazovaya diagramma sistemy $\mathrm{NiO}-\mathrm{MoO}_{3}$. [Phase diagram of the system $\mathrm{NiO}-\mathrm{MoO}_{3}$ ]. Russ J Inorg Chem. 1974;19(7):1932-6.

11. Zhukovsky VM, Tkachenko EV. Fazovye ravnovesiya v molibdatnykh sistemakh. [Phase equilibria in molybdate systems]. Zbornik nauchnykh trudov. "Fizicheskaya khimiya okislov". Moscow: Nauka; 1981.106-15. Russian.

12. Reichelt W, Weber T, Soehnel T. at al. Electronic structure and luminescence mechanisms in $\mathrm{ZnMoO}_{4}$ crystals. J Phys: Condes Mater. 2011;23:244-59.

13. Tsyrenova GD, Bazarova JG, Mokhosoev MV. Phase equilibria in systems MeO$\mathrm{MoO}_{3}$ (Me-Mg, Мп, Zn). Russ J Inorg Chem. 1986;31(12):3120-3. 
14. Rajaram P, Viswanathan B, Aravamudan G. Studies on the formation of manganese molybdates. Thermochim acta. 1973;7 (2):123-9.

15. Kohlmuller R, Faurie J.-P. Etude des systemes $\mathrm{MoO}_{3}-\mathrm{Ag}_{2} \mathrm{MoO}_{4}$ et $\mathrm{MoO}_{3}-\mathrm{MO}(\mathrm{M}-\mathrm{Cu}$, Zn, Cd). Bull Soc chim. France. 1968;11:4379-82.

16. Larson AC, Von Dreele RB. General Structure Analysis System (GSAS) (Report LAUR 86-748). 2004. Los Alamos, New Mexico: Los Alamos National Laboratory.

17. Shannon RD. Revised effective ionic radii and systematic studies of interatomic distances in dalides and chalcogenides. Acta Cryst. 1976; A32:751-67. DOI: $10.1107 /$ S0567739476001551.

18. Gates Stacy D, Julie A, Lind C. Gates Non-hydrolytic sol-gel synthesis, properties, and high-pressure behavior of gallium molybdate. J Mater Chem. 2006;16:4214-9. DOI:10.1039/B608864C.

19. Perepelitsa AP, Golub AM, Badayev YuB, Shapoval VN. Double molybdates of aluminum, gallium, indium, chromium, iron and bismuth with monovalent silver and thallium. Russ J Inorg Chem. 1977;22(4):994-7.

20. Klevtsova RF, Vasiliev AD, Kozhevnikova NM, Glinskaya LA, Kruglik AI, Kotova IYu. Synthesis and crystal structural study of ternary molybdate $\mathrm{NaMg}_{3} \mathrm{In}\left(\mathrm{MoO}_{4}\right)_{5}$. J Struct Chem. 1994;34:784-8.

21. Rietveld HM. A profile refinement method for nuclear and magnetic structures. J Appl Cryst. 1969;2:65-71. 